Tohoku J. Exp. Med., 2010, 220, 307-318

\title{
Community-Based Lifestyle Modification of Cardiovascular Disease Risks in Middle-Aged Japanese: A 27-Month Update
}

\author{
Hiroko Fujii, ${ }^{1}$ Takashi Muto, ${ }^{1}$ Yasuo Haruyama, ${ }^{1}$ Makiko Nakade, ${ }^{2}$ \\ Emiko Kobayashi, ${ }^{3}$ Kaori Ishisaki ${ }^{3}$ and Akiko Yamasaki ${ }^{3}$ \\ ${ }^{1}$ Department of Public Health, Dokkyo Medical University School of Medicine, Shimotsuga, Tochigi, Japan \\ ${ }^{2}$ Nutritional Education Program, National Institute of Health and Nutrition, Shinjuku, Tokyo, Japan \\ ${ }^{3}$ Soka City Health Center, Soka, Saitama, Japan
}

\begin{abstract}
Lifestyle modification is the cornerstone of preventive management for people with cardiovascular disease risks, such as obesity, hypertension, dyslipidemia and diabetes. This study investigated the effect of a 27-month community-based lifestyle intervention on the reduction of cardiovascular disease risks in middle-aged Japanese. Of 549 participants with cardiovascular disease risk factors of overweight, hypertension, dyslipidemia or diabetes enrolled in this non-randomized controlled study, 397 participants aged 39-71 years old completed all 3 serial surveys at baseline, 15 months and 27 months. For the intervention group (39 males and 174 females), 31 specific interventions including individual counselling and group sessions were conducted. The control group (64 males and 120 females) only received 7 newsletters providing health information and results of health checkups. Multiple logistic regression analysis adjusted for sex, each baseline risk category and age category showed that the proportion of those who were overweight or showed dyslipidemia risk were significantly lower in the intervention group only at 27 months [Odds ratio (OR): $0.43(95 \% \mathrm{Cl} 0.20-0.94)$, OR: $0.43(95 \% \mathrm{Cl} 0.21-0.87)$, respectively] and the proportion of those showing diabetes risk was significantly lower in the intervention group at both 15 months [OR: $0.42(95 \% \mathrm{Cl}$ 0.18-0.97)] and 27 months [OR: 0.56 (95\% Cl 0.32-0.99)]. In conclusion, the 27-month community-based lifestyle modification of cardiovascular disease risks shows significant reductions in risks of diabetes, overweight and dyslipidemia in middle-aged Japanese.
\end{abstract}

Keywords: cardiovascular disease; lifestyle intervention; community; diet; physical activity

Tohoku J. Exp. Med., 2010, 220 (4), 307-318. (C) 2010 Tohoku University Medical Press

Lifestyle-related diseases such as hypertension, dyslipidemia, diabetes and obesity are major risk factors of cardiovascular disease (Sakuma and Kitabatake 2008). Heart disease is the second leading cause of mortality in Japan (Health and Welfare Statistic Association 2008). An increased number of patients with cardiovascular disease including ischemic heart disease, such as angina pectoris and myocardial infarction, is anticipated due to an increase in atherosclerosis with aging (Sakuma and Kitabatake 2008). Lifestyle modification, which is the cornerstone of preventive management for people with these risk factors, should be recommended (Committee on Guideline for Treatment of Obesity 2006; Japan Atherosclerosis Society 2007; Japan Diabetes Society 2008; Committee on Guideline for the Management of Hypertension 2009).

Previous studies have shown that lifestyle modification can reduce the incidence of diabetes (Knowler et al. 2002; Lindstrom et al. 2003; Lindstrom et al. 2006; Gillies et al. 2007), hypertension (Appel et al. 2003; Elmer et al. 2006) and cardiovascular disease risk factors (Ratner et al. 2005; Maruthur et al. 2009), and the development of effective and feasible health education programs in community settings contributes to reducing these risks. In a Japanese community, the use of existing health examination in community health services as a basis for lifestyle intervention programs is considered one of the most feasible methods (Egawa et al. 2004). Several trials in community settings have demonstrated the effect of lifestyle interventions targeting a middle-aged community-dwelling population for the reduction of obesity (Mitsuhashi et al. 2003; Noda et al. 2006; Egawa et al. 2007), hypertension (Iso et al. 1996; Miura et al. 2006), diabetes (Kuriyama et al. 2006), dyslipidemia (Amano et al. 2002; Iso et al. 2002) and risk of cardiovascular diseases (Haruyama et al. 2009). Using data of the same cohort as the present study, Haruyama et al. (2009) found that there was a significant mean weight reduction and increase of physical activity in a lifestyle intervention group compared with a control group for 15 months among males

Received November 18, 2009; revision accepted for publication March 12, 2010. doi:10.1620/tjem.220.307

Correspondence: Hiroko Fujii, Department of Public Health, Dokkyo Medical University School of Medicine, 880 Kitakobayashi, Mibu,

Shimotsuga, Tochigi, 321-0293, Japan.

e-mail: hirofuji@dokkyomed.ac.jp 
and females. Though most of these studies evaluated programs lasting 3 to 18 months and showed improvements in each target risk (Iso et al. 1996, 2002; Amano et al. 2002; Mitsuhashi et al. 2003; Kuriyama et al. 2006; Miura et al. 2006; Noda et al. 2006; Egawa et al. 2007), the effect of lifestyle intervention programs for two years or longer in Japanese community settings have not yet been clarified. We consider that it is important to evaluate the effects of programs conducted over longer periods in community settings in order to obtain a more appropriate evaluation aimed at future policy-making.

The present study investigated whether a long-term intervention program conducted by local community health workers targeting a high risk population in a community setting could achieve desirable effects on lifestyle behaviors and cardiovascular disease risks at 27 months on further follow-up of subjects from the previous study (Haruyama et al. 2009).

\section{Methods}

\section{Study design and participants}

Details of the project in the present study have been reported previously (Haruyama et al. 2009). The lifestyle intervention program was designed by local community health workers in Soka city based on the "Health-Up Model Project" by the Ministry of Health, Labour and Welfare of Japan (Haruyama et al. 2009). The project staff included doctors at a local hospital, public health nurses and registered dietitians at a health center and instructors at a sports association in the city (Haruyama et al. 2009). We newly examined the effect of the program over a longer term, 27 months.

The program was provided between July 2004 and September 2006. In 2003, 17,395 residents of Soka city underwent health checkup for the elderly conducted by the municipality. A total of 7,875 residents aged 39-71 years old, who had at least one risk factor of overweight, hypertension, dyslipidemia or diabetes in the health checkup (Ministry of Health, Labour and Welfare of Japan 2005a), were informed of the study by direct mail. We used the same inclusion categories as the previously published study (Haruyama et al. 2009). Namely, of the 636 subjects recruited, 549 were included in this study because they were considered one of the following types of high-risk subjects. Subjects with overweight were defined as having a body mass index $(\mathrm{BMI}) \geq 25 \mathrm{~kg} / \mathrm{m}^{2}$; subjects with hypertension risk were defined as having at least one of the following: systolic blood pressure $(\mathrm{SBP}) \geq 130 \mathrm{mmHg}$ or diastolic blood pressure $(\mathrm{DBP}) \geq 85 \mathrm{mmHg}$; subjects with dyslipidemia risk were defined as having at least one of the following: total cholesterol (TC) $\geq 200 \mathrm{mg} / \mathrm{dl}$ (females aged 50 years or over: $\geq 220 \mathrm{mg} / \mathrm{dl}$ ), high-density lipoprotein cholesterol (HDL-C) $<40 \mathrm{mg} / \mathrm{dl}$, low-density lipoprotein cholesterol (LDL-C) $\geq 120 \mathrm{mg} / \mathrm{dl}$ or triglycerides (TG) $\geq 150 \mathrm{mg} / \mathrm{dl}$ and subjects with diabetes risk were defined as having at least one of the following: fasting plasma glucose $(\mathrm{FPG}) \geq 110 \mathrm{mg} / \mathrm{dl}$ or hemoglobin $\mathrm{A}_{1 \mathrm{c}}\left(\mathrm{HbA}_{1 \mathrm{c}}\right) \geq 5.6 \%$. We defined the inclusion category at baseline with reference to the risk criteria for Cardiovascular Disease in Health Care of Aged (Japan Public Health Association, 2002), Adult Treatment Panel III criteria, and the National Cholesterol Education Program (NCEP-ATP III 2002), Japan Atherosclerosis Society (Japan Atherosclerosis Society 2007) and report on the survey of diabetes (Ministry of Health, Labour and Welfare 2004). All subjects self-reported that they were not taking any medication prescribed for any abnormality with verification by their local physicians. Individuals were advised by doctors to be seen at medical institutions, if necessary.

As shown in Fig. 1, a total of 549 participants enrolled in this non-randomized controlled study. These participants were allocated to either the intervention or the control group based on each participant's own preference. Of these participants, 397 participants completed all 3 assessments at baseline, 15 months, and 27 months. Ultimately, the results of 397 (drop-out rate: 27.7\%) participants, consisting of 213 participants in the intervention group (39 males and 174 females) and 184 participants in the control group (64 males and 120 females), were analyzed.

The study protocol was approved by the Soka City Board. Then the study protocol was submitted by the Soka City Board to the Ministry of Health, Labour and Welfare of Japan, where it was also approved. Written informed consent to participate in this study, which included an explanation of the risks of performing exercise, was obtained from each subject. The Soka City Board entrusted the analysis to researchers at Dokkyo Medical University. Data were unlinkably anonymized before being sent to the researchers at Dokkyo Medical University in charge of the analysis. Therefore, all personal information has been protected, and the study protocol is in compliance with the Ethical Guidelines for Epidemiological Research (Ministry of Education, Culture, Sports, Science and Technology, and Ministry of Health, Labour and Welfare, Japan, 2002).

\section{Intervention}

The intervention group attended 2 individual counselling sessions for 1 hour each and 15 group sessions for 2 hours each during the initial 15 months. As shown in Table 1, group sessions during the first 6 months were more frequent than those during the next 9 months. In addition, the intervention group attended one individual counselling session and 13 group sessions held during the following 12 months.

Individual counselling was based on the results of health checkups and health assessment of physical activity, dietary intake, and other lifestyle habits at each time point. The group sessions on nutrition by registered dietitians consisted of lectures on appropriate diet for preventing lifestyle-related disease, such as increasing vegetable intake for dietary fiber, vitamins and minerals, preferable fat intake and decreased salt intake, and cooking classes on how to prepare food appropriately.

The group exercise sessions conducted by exercise instructors, consisted activities such as aerobic exercises, stretching, walking, and water-based exercise in a sports gym or some local health and community centers. Participants received a medical check before starting the exercise program, and their conditions were confirmed by exercise instructors at each session. Participants in the intervention group were divided into 9 small groups (30 to 35 people in each group), and the same program was provided during the daytime and at night for the convenience of participants. Through these approaches, participants were encouraged to set their own goals for lifestyle modification.

Moreover, newsletters were delivered to the intervention group and the control group for 27 months, as shown in Table 1. The intervention group received newsletters providing information on health and the control group received newsletters with the results of their health checkups and other information on health.

The mean attendance rates in the intervention group were $86.6 \%$ during the initial 15 months and $74.4 \%$ during the following 12 


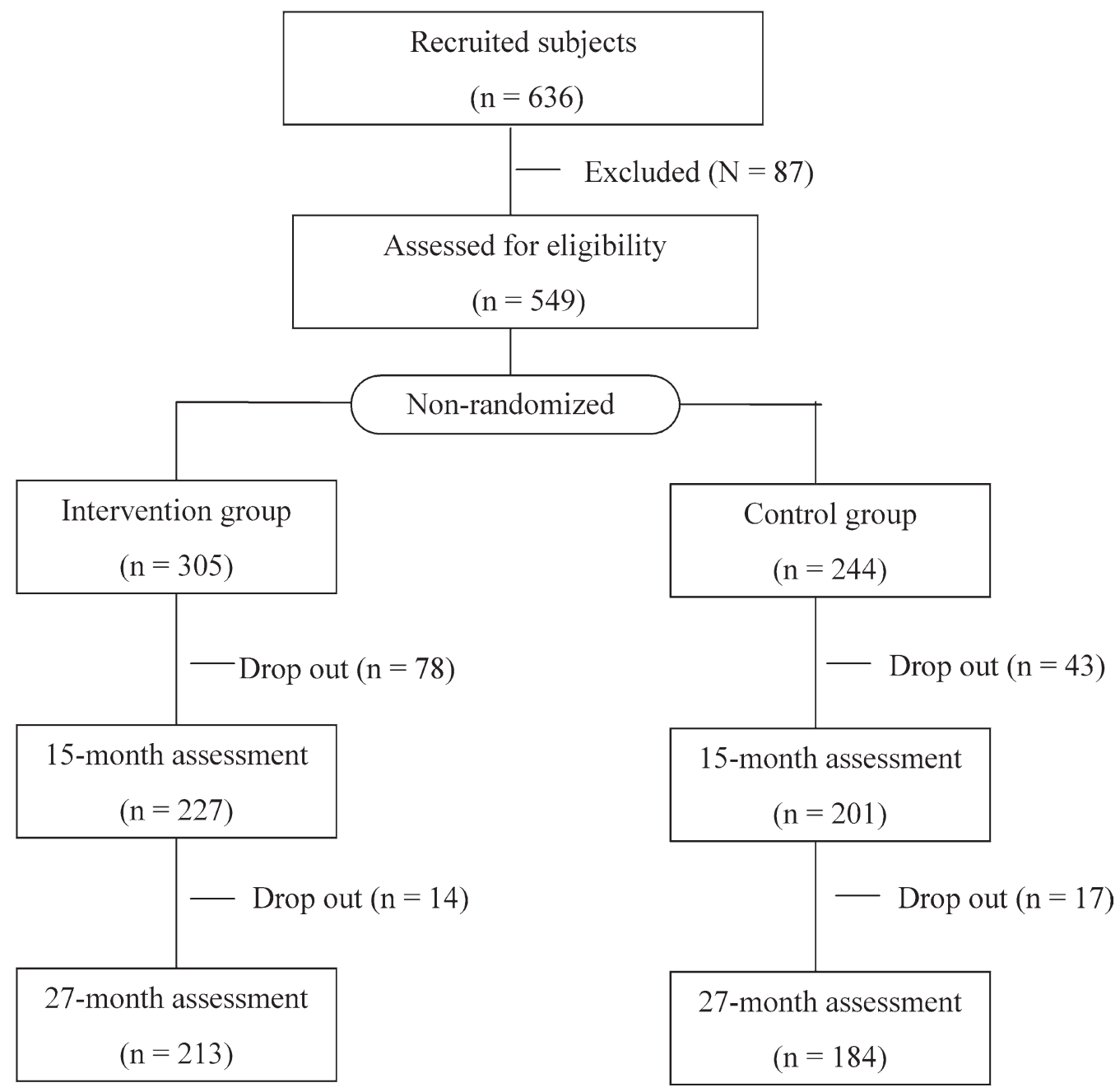

Fig. 1. Flowchart of the study protocol.

Table 1. The contents of the programs for the intervention group and the control group.

\begin{tabular}{|c|c|c|c|}
\hline & & Intervention group & Control group \\
\hline \multirow[t]{12}{*}{ Initial 15 months } & First 6 months & Health checkup ${ }^{\mathrm{a}}$ & Health checkup ${ }^{\mathrm{a}}$ \\
\hline & & Individual counseling ( 1 hour, 1 time) & Newsletter (3 times) \\
\hline & & Group session ( 2 hours, 11 times) & \\
\hline & & Nutrition (2 times) & \\
\hline & & Exercise (9 times) & \\
\hline & & Newsletter (3 times) & \\
\hline & Next 9 months & Health checkup & Health checkup \\
\hline & & Individual counseling ( 1 hour, 1 time) & Newsletter (2 times) \\
\hline & & Group session ( 2 hours, 4 times) & \\
\hline & & Nutrition (1 time) & \\
\hline & & Exercise (3 times) & \\
\hline & & Newsletter (2 times) & \\
\hline \multirow[t]{6}{*}{ Following 12 months } & & Health checkup ${ }^{\mathrm{b}}$ & Health checkup ${ }^{b}$ \\
\hline & & Individual counseling ( 1 hour, 1 time) & Newsletter (2 times) \\
\hline & & Group session ( 2 hours, 13 times) & \\
\hline & & Nutrition (4 times) & \\
\hline & & Exercise (9 times) & \\
\hline & & Newsletter (2 times) & \\
\hline
\end{tabular}

a. Health checkup for baseline assessment.

b. Health checkup for 15-month assessment. 
months.

\section{Measurements}

Body weight was measured, while subjects were lightly clothed without shoes using the TBF110 body fat monitor (Tanita Inc., Tokyo, Japan). Height was measured using the height measuring scale with a handle VL65 (Yagami Inc., Nagoya, Japan) without shoes. BMI was calculated as body weight $(\mathrm{kg})$ divided by the square of the height $\left(\mathrm{m}^{2}\right)$. SBP and DBP were measured by the oscillometric methods using auto-manometers HEM 762 Fuzzy (Omron Co., Tokyo, Japan) after the subjects had been seated for 3-5 minutes. If the first measurement value was high, it was taken again after the subjects had remained still for 3 minutes, and the lower value was recorded (Sairenchi et al. 2005). Fasting blood samples from all subjects were obtained and TC, HDL-C, LDL-C, TG, FPG and $\mathrm{HbA}_{1 \mathrm{c}}$ were measured at a laboratory (Saitama, Japan). For the determination of each risk category of evaluations at both 15 months and 27 months, we included selfreported medication usage for the treatment of hypertension, dyslipidemia or diabetes, referring to the determination of subjects with metabolic syndrome risk category in the national health and nutrition survey (Ministry of Health, Labour and Welfare 2009).

We used the Framingham risk score, which is based on age, TC, HDL-C, SBP and current smoking status (Expert Panel on Detection, Evaluation, and Treatment of High Blood Cholesterol in Adults 2001), in order to establish risk scores. TC was classified into 5 levels $(<160,160-199,200-239,240-279, \geq 280 \mathrm{mg} / \mathrm{dl})$, HDL-C was classified into 4 levels ( $\geq 60,50-59,40-49,<40 \mathrm{mg} / \mathrm{dl}$ ) and SBP was classified 5 levels $(<120,120-129,130-139,140-159, \geq 160 \mathrm{mmHg})$.

\section{Food frequency questionnaire and dietary intake}

We evaluated dietary intake of the participants during the previous 1-2 months using the food frequency questionnaire (FFQ) and the software package Excel Eiyoukunn; the FFQ was based on the major food groups (Kenpakusya Corp., Tokyo, Japan) (Yoshimura and Takahashi 2001). Detailed descriptions of the methods used for calculating dietary intake and the validity of the FFQ have been published elsewhere (Takahashi 2003). Pearson correlation coefficients between FFQ and 7-day dietary records were 0.47 for energy, 0.42 for protein, 0.39 for fat, 0.49 for carbohydrate, 0.44 for dietary fiber and 0.43 for salt (Takahashi, 2003).

In the questionnaire, participants also self-reported the daily duration of the classification of 5 physical activity; sleeping, highintensity activities (e.g., jogging, carrying a heavy load), moderateintensity activities (e.g., walking at a moderate pace, bicycling at a moderate pace), low-intensity activities (e.g., walking slowly, light cleaning), and sedentary activities and standing quietly (e.g., sitting while reading a book, light desk work, talking, eating). Activity factors (Af) of the 5 physical activities, which indicated the intensity of each physical activity, were expressed in a multiple of the basal metabolism and used to estimate physical activity level for each subject (Ministry of Health, Labour and Welfare of Japan 2005b, Sasaki 2007). The calculated physical activity level (Af) was used as each subject's physical activity level (Af) to assess individual energy intake because the estimated energy requirement of each adult subjects is defined as "the energy intake that is estimated to have the highest probability of achieving an energy balance (energy expenditure-energy intake) of zero" (Ministry of Health, Labour and Welfare of Japan 2005b; Sasaki 2007).

We confirmed that there were no participants who had extremely low or high energy intake estimated by the FFQ (range of energy intake: 855-3901 kcal).

Nutrient intakes in this study were energy adjusted using energy density; intake of fat, protein, carbohydrate by energy percent (E\%), dietary fiber and salt by intake (g) per 1,000 kcal energy intake, showing dietary fiber intake and salt intake, respectively. In this study, preferable nutrient intake were defined as follows: fat intake (E\%) $\geq 20$ and $<25$, carbohydrate intake $(\mathrm{E} \%) \geq 50$ and $<70$, dietary fiber intake $\geq 10 \mathrm{~g} / 1,000 \mathrm{kcal}$ and salt density $<4.5 \mathrm{~g} / 1,000 \mathrm{kcal}$, referring to the nutrition targets in the guidelines for primary prevention of ischemic heart disease (Sakuma and Kitabatake 2008) and dietary reference intakes for Japanese (Ministry of Health, Labour and Welfare of Japan 2005b).

\section{Assessment of lifestyle factors}

Information on lifestyles such as current medication usage, smoking, alcohol drinking, eating behaviors, and physical activity were obtained by a self-administered questionnaire. Smoking status was indicated using three categories: current smoker, ex-smoker and never smoked. Drinking status was confirmed by the frequency of drinking and quantity of alcohol consumption. Habitual drinker was defined as one who takes more than approximately $20 \mathrm{~g}$ of alcohol per day for 3 days or more per week. Dietary behaviors or current medication usage were categorized as yes or no. Preferable dietary behaviors included eating slowly, not eating until full and not habitually eating snacks, based on the assumption that these dietary behavior are associated with risk factors of cardiovascular disease (Otsuka et al. 2006, 2008; Maruyama et al. 2008; Woo et al. 2008). A regular exerciser was defined as a participant with an exercise frequency of 20 minutes sessions more than 2 times per week. Number of walking steps was self-reported using a pedometer distributed to each subject at baseline. Walking more than 6,000 steps per day was defined as preferable.

\section{Statistical analysis}

We selected lifestyle behaviors and cardiovascular disease risks as outcome measures in this study. The lifestyle behaviors included physical activity, dietary behaviors and nutrient intakes. The cardiovascular disease risks included overweight, hypertension, dyslipidemia and diabetes. These two outcome measures each have benefits and limitations. Cardiovascular disease risks are more objective than lifestyle behaviors, but they are influenced by genetic factors. Lifestyle behaviors are subjective, but they are more direct measures of lifestyle intervention.

Comparison between the intervention group and the control group at baseline were examined by Student's $t$-test for continuous variable of anthropometric and biological data, total risk score energy intake and physical activity level, and by Chi-squared test or Fisher's exact test for categorical variables of disease risk, lifestyle behavior and smoking status.

We performed an analysis of variance for repeated measures to determine time-related change in anthropometric and biological data at 15 months and 27 months among subjects at each risk. To remove the influence of medications on changes in anthropometric and biological data, we excluded subjects who self-reported that they were taking medication prescribed for hypertension (9 males and 23 females), dyslipidemia ( 2 males and 22 females) and diabetes ( 3 males and 3 females) in this analysis of variance for repeated measures. In the analysis, we included all 394 subjects in terms of the 
Framingham risk score, energy intake and physical activity level.

We analyzed the probability of subjects with preferable lifestyle factors or cardiovascular disease risks being in the intervention group versus the control group using multiple logistic regression analysis. In conducting multiple logistic analysis, the dependent variable was lifestyle behavior (0: Not the preferable lifestyle behavior, 1: Preferable lifestyle behavior) and cardiovascular disease risks (0: Without risks, 1: With risks). For independent variables, we defined the group allocation (0: Control group, 1: Intervention group), each baseline category and sex (0: male, 1 : female), age category (0: 39-49 years, 1: 50-59 years, 2: 60-71 years), and baseline category of each lifestyle behavior or each cardiovascular disease risk. We employed two models: model 1 adjusted for age category and each baseline category for male and female participants, respectively: model 2 adjusted for sex, age category and each baseline category for all participants. Odds ratios (OR) with $95 \%$ confidence intervals (CI) were calculated in terms of prevalence of preferable lifestyle behaviors in the intervention group against that in the control group and prevalence of cardiovascular disease risks in the intervention group against that in the control group.

For all tests, a value of $P<0.05$ was considered significant. All analyses were performed using the SPSS 15.0J for Windows (SPSS Japan Inc., Tokyo, Japan).

\section{Results}

\section{Baseline characteristics of participants}

Table 2 shows the comparison of the baseline lifestyle factors between the intervention group and the control group. The proportion of those with preferable carbohydrate intake was significantly higher in the intervention group than that in the control group for males $(P=0.028)$. Table 3 shows the comparison of the baseline cardiovascular disease risks between the two groups. The proportion of overweight females in the intervention group was significantly higher than that in the control group $(P=0.015)$. There were no other significant differences in baseline characteristics between the two groups among males and females.

\section{Changes in lifestyle behaviors}

Table 4 shows the odds ratios of preferable lifestyle behaviors in the intervention group versus the control group. Multiple logistic regression analysis adjusted for each baseline category and age category showed that the proportion of males performing regular exercise was significantly

Table 2. Comparison of the baseline lifestyle factors between the intervention group and the control group.

\begin{tabular}{|c|c|c|c|c|c|c|}
\hline & \multicolumn{3}{|c|}{ Males } & \multicolumn{3}{|c|}{ Females } \\
\hline & Intervention & Control & $P$-value ${ }^{\mathrm{a}}$ & Intervention & Control & $P$-value ${ }^{\mathrm{a}}$ \\
\hline$n$ & 39 & 64 & & 174 & 120 & \\
\hline Age, mean \pm S.D. & $60.7 \pm 9.2$ & $62.2 \pm 8.1$ & $0.407^{\mathrm{b}}$ & $62.2 \pm 5.8$ & $60.9 \pm 7.0$ & $0.086^{\mathrm{b}}$ \\
\hline \multicolumn{7}{|l|}{ Age category, $n(\%)$} \\
\hline 39-49 years & $6(15.4)$ & $8(12.5)$ & $0.887^{\mathrm{c}}$ & $4(2.3)$ & $10(8.3)$ & $0.142^{\mathrm{c}}$ \\
\hline $50-59$ years & $3(7.7)$ & $6(9.4)$ & & $35(20.1)$ & $26(21.7)$ & \\
\hline $60-71$ years & $30(76.9)$ & $50(78.1)$ & & $135(77.6)$ & $84(70.0)$ & \\
\hline Current smoker, $n(\%)$ & $12(30.8)$ & $16(25.0)$ & 0.523 & $11(6.3)$ & $6(5.0)$ & 0.633 \\
\hline \multicolumn{7}{|l|}{ Physical activity } \\
\hline Regular exerciser, $n(\%)$ & $21(53.8)$ & $29(45.3)$ & 0.401 & $84(48.3)$ & $50(41.7)$ & 0.263 \\
\hline Walking steps $\geq 6,000 /$ day & $25(64.1)$ & $44(68.8)$ & 0.627 & $108(62.1)$ & $87(72.5)$ & 0.063 \\
\hline \multicolumn{7}{|l|}{ Dietary behavior } \\
\hline Eating slowly, yes, $n(\%)$ & $20(51.3)$ & $30(46.9)$ & 0.664 & $92(52.9)$ & $67(55.8)$ & 0.617 \\
\hline Not eating until full, yes, $n(\%)$ & $28(71.8)$ & $45(70.3)$ & 0.872 & $111(63.8)$ & $87(72.5)$ & 0.118 \\
\hline Habitually eating snacks, no, $n(\%)$ & $25(64.1)$ & $46(71.9)$ & 0.408 & $79(45.4)$ & $42(35.0)$ & 0.075 \\
\hline Not Habitual drinker, $n(\%)$ & $13(33.3)$ & $15(23.4)$ & 0.274 & $114(65.5)$ & $69(57.5)$ & 0.163 \\
\hline \multicolumn{7}{|l|}{ Nutrient intake and physical activity level } \\
\hline Energy, kcal, mean \pm S.D. & $2,149 \pm 482$ & $1,995 \pm 499$ & $0.124^{\mathrm{b}}$ & $1,845 \pm 355$ & $1,823 \pm 363$ & $0.615^{b}$ \\
\hline Physical activity level, Af, mean \pm S.D. & $1.6 \pm 0.2$ & $1.5 \pm 0.1$ & $0.616^{\mathrm{b}}$ & $1.6 \pm 0.2$ & $1.7 \pm 0.1$ & $0.448^{b}$ \\
\hline Fat $(\mathrm{E} \%) \geq 20$ and $<25, n(\%)$ & $8(20.5)$ & $16(25.0)$ & 0.601 & $39(22.4)$ & $25(20.8)$ & 0.747 \\
\hline Carbohydrate $(\mathrm{E} \%) \geq 50$ and $<70, n(\%)$ & $38(97.4)$ & $53(82.8)$ & $0.028^{\mathrm{d}}$ & $169(97.1)$ & $110(91.7)$ & $0.056^{\mathrm{d}}$ \\
\hline Dietary fiber $\geq 10 \mathrm{~g} / 1,000 \mathrm{kcal}, n(\%)$ & $2(5.1)$ & $4(6.3)$ & $1.000^{\mathrm{d}}$ & $28(16.1)$ & $21(17.5)$ & 0.750 \\
\hline Salt $<4.5 \mathrm{~g} / 1,000 \mathrm{kcal}, n(\%)$ & $10(25.6)$ & $12(18.8)$ & 0.408 & $27(15.5)$ & $21(17.5)$ & 0.651 \\
\hline
\end{tabular}

E\%, Energy percent; g/1,000 kcal, g per 1,000 kcal energy intake; Af, Activity factor.

a. $P$ value was determined by Chi-squared test, unless otherwise specified.

b. $P$ value was determined by unpaired Student's $t$-test.

c. $P$ value was determined by Chi-squared test in the two age categories: age $<59$ years and age $\geq 60$ years or over.

d. $P$ value was determined by Fisher's exact test. 
Table 3. Comparison of baseline cardiovascular disease risks between intervention and control group.

\begin{tabular}{|c|c|c|c|c|c|c|}
\hline & \multicolumn{3}{|c|}{ Males } & \multicolumn{3}{|c|}{ Females } \\
\hline & Intervention & Control & $P$-value ${ }^{\mathrm{b}}$ & Intervention & Control & $P$-value ${ }^{\mathrm{b}}$ \\
\hline & Mean \pm S.D. & Mean \pm S.D. & & Mean \pm S.D. & Mean \pm S.D. & \\
\hline$n$ & 39 & 64 & & 174 & 120 & \\
\hline Weight & $67.6 \pm 9.2$ & $64.4 \pm 10.0$ & 0.111 & $54.4 \pm 8.3$ & $53.7 \pm 8.7$ & 0.461 \\
\hline BMI, $\mathrm{kg} / \mathrm{m}^{2}$ & $24.8 \pm 2.5$ & $23.7 \pm 3.0$ & 0.067 & $23.7 \pm 3.5$ & $23.0 \pm 3.4$ & 0.061 \\
\hline Overweight $^{\mathrm{e}}, n(\%)$ & $18(46.2)$ & 19 (29.7) & $0.091^{\mathrm{c}}$ & $64(36.8)$ & $28(23.3)$ & $0.015^{\mathrm{c}}$ \\
\hline $\mathrm{SBP}, \mathrm{mmHg}$ & $140.7 \pm 10.4$ & $138.1 \pm 14.3$ & 0.292 & $135.4 \pm 16.6$ & $135.5 \pm 17.6$ & 0.974 \\
\hline DBP, mmHg & $82.6 \pm 9.0$ & $83.1 \pm 10.4$ & 0.825 & $79.3 \pm 10.1$ & $79.0 \pm 10.7$ & 0.832 \\
\hline Hypertension risk $^{\mathrm{f}}, n(\%)$ & $31(79.5)$ & $48(75.0)$ & $0.601^{\mathrm{c}}$ & $115(66.1)$ & $80(66.7)$ & $0.918^{\mathrm{c}}$ \\
\hline $\mathrm{TC}, \mathrm{mg} / \mathrm{dl}$ & $206.6 \pm 24.9$ & $207.4 \pm 28.6$ & 0.886 & $229.9 \pm 34.8$ & $237.2 \pm 31.7$ & 0.066 \\
\hline HDL-C, mg/dl & $54.6 \pm 14.0$ & $54.4 \pm 14.7$ & 0.949 & $66.5 \pm 15.3$ & $67.6 \pm 14.7$ & 0.547 \\
\hline LDL-C, mg/dl & $136.5 \pm 22.0$ & $130.2 \pm 28.1$ & 0.231 & $147.5 \pm 34.6$ & $152.3 \pm 30.9$ & 0.221 \\
\hline $\mathrm{TG}, \mathrm{mg} / \mathrm{dl}$ & $105.0 \pm 41.8$ & $119.9 \pm 68.1$ & 0.372 & $94.8 \pm 44.8$ & $101.5 \pm 48.0$ & 0.149 \\
\hline Dyslipidemia risk $^{\mathrm{g}}, n(\%)$ & $36(92.3)$ & $51(79.7)$ & $0.100^{\mathrm{d}}$ & $154(88.5)$ & $108(90.0)$ & $0.686^{\mathrm{c}}$ \\
\hline $\mathrm{FPG}, \mathrm{mg} / \mathrm{dl}$ & $101.4 \pm 12.0$ & $101.8 \pm 13.2$ & 0.866 & $98.7 \pm 20.5$ & $98.0 \pm 14.9$ & 0.732 \\
\hline $\mathrm{HbA}_{1 \mathrm{c}}, \%$ & $5.24 \pm 0.50$ & $5.23 \pm 0.58$ & 0.927 & $5.24 \pm 0.64$ & $5.26 \pm 0.53$ & 0.742 \\
\hline Diabetes risk $^{\mathrm{h}}, n(\%)$ & $9(23.1)$ & $20(31.3)$ & $0.371^{\mathrm{c}}$ & $35(20.1)$ & $21(17.5)$ & $0.575^{\mathrm{c}}$ \\
\hline Framingam risk score, score & $12.2 \pm 2.7$ & $12.3 \pm 2.9$ & 0.818 & $14.3 \pm 2.8$ & $14.2 \pm 2.8$ & 0.858 \\
\hline
\end{tabular}

BMI, Body mass index; SBP, Systolic blood pressure; DBP, Diastolic blood pressure; TC, Total cholesterol; HDL-C, High-density lipoprotein cholesterol; LDL-C, Low-density lipoprotein cholesterol; TG, Triglycerides; FPG, Fasting plasma glucose; HbA Hemoglobin $\mathrm{A}_{1 \mathrm{c}}$.

a. Values are shown as mean \pm S.D., unless otherwise specified.

b. $P$ value was determined by unpaired Student's $t$-test, unless otherwise specified

c. $P$ value was determined by Chi-squared test.

d. $P$ value was determined by Fisher's exact test.

e. Subjects with overweight were defined as having a BMI $\geq 25 \mathrm{~kg} / \mathrm{m}^{2}$.

f. Subjects with hypertension risk were defined as having at least one of the following: $\mathrm{SBP} \geq 130 \mathrm{mmHg}$ or DBP $\geq 85 \mathrm{mmHg}$.

g. Subjects with dyslipidemia risk were defined as having at least one of the following: TC $\geq 200 \mathrm{mg} / \mathrm{dl}$ (females aged 50 years or over: $220 \mathrm{mg} / \mathrm{dl}$ ), HDL-C $<40 \mathrm{mg} / \mathrm{dl}$, LDL-C $\geq 140 \mathrm{mg} / \mathrm{dl}$ or TG $\geq 150 \mathrm{mg} / \mathrm{dl}$.

h. Subjects with diabetes risk were defined as having at least one of the following: $\mathrm{FPG} \geq 110 \mathrm{mg} / \mathrm{dl}$ or $\mathrm{HbA}_{1 \mathrm{c}} \geq 5.6 \%$.

higher in the intervention group than those in the control group only at 15 months (OR 5.68, CI 1.85-17.42). In females, the proportion of those who had preferable fat intake was significantly increased in the intervention group at 15 months (OR 2.39, CI 1.26-4.55) and 27 months (OR 1.95, CI 1.04-3.66). The proportions of females consuming a preferable carbohydrate intake and dietary fiber intake were significantly greater in the intervention group than those in the control group only at 15 months (OR 2.93, CI 1.04-8.25 and OR 2.12, CI 1.12-4.03, respectively) and the proportion of females performing regular exercise or those walking a preferable number of steps was significantly greater in the intervention group only at 15 months (OR 2.38, CI 1.35-4.19 and OR 4.17, CI 2.10-8.27, respectively). In addition, the proportion of females having a habit of not eating until full was significantly greater in the intervention group only at 27 months (OR 2.95, CI 1.45-5.99).

Multiple logistic regression analysis adjusted for sex, each baseline risk category and age category by sex showed that the proportion of those walking a preferable number of steps was significantly greater in the intervention group at both 15 months (OR 3.80, CI 2.07-6.97) and 27 months (OR 1.90, CI 1.07-3.37). However, the proportion of those performing regular exercise was significantly higher in the intervention group than those in the control group only at 15 months (OR 2.89, CI 1.75-4.76). The proportion of those consuming a preferable dietary fiber intake was significantly greater in the intervention group than in the control group only at 15 months (OR 2.17, CI 1.20-3.90) and the proportion of those having a habit of not eating until full was significantly greater in the intervention group only at 27 months (OR 2.32, CI 1.29-4.18). There were no significant differences in energy intake or physical activity level throughout the 27 months among males or females in the two groups, respectively (data are not shown). 
Table 4. Odd ratios of preferable lifestyle behaviors in intervention vs. control group.

\begin{tabular}{|c|c|c|}
\hline & Intervention vs. Control at 15 months & Intervention vs. Control at 27 months \\
\hline & OR $(95 \% \mathrm{CI})^{\mathrm{a}}$ & OR $(95 \% \mathrm{CI})^{\mathrm{a}}$ \\
\hline Males, $n$ & 39 vs. 64 & 39 vs. 64 \\
\hline \multicolumn{3}{|l|}{ Physical activity } \\
\hline Regular exerciser & $5.68(1.85-17.42)$ & $1.68(0.62-4.52)$ \\
\hline Walking steps $\geq 6,000 /$ day & $4.09(0.98-17.07)$ & $1.92(0.57-6.39)$ \\
\hline \multicolumn{3}{|l|}{ Dietary behavior } \\
\hline Eating slowly, yes & $0.93(0.35-2.49)$ & $0.91(0.33-2.51)$ \\
\hline Not eating until full, yes & $1.92(0.56-6.56)$ & $1.34(0.44-4.06)$ \\
\hline Habitually eating snacks, no & $0.58(0.21-1.62)$ & $1.29(0.50-3.28)$ \\
\hline Not Habitual drinker & $1.00(0.24-4.21)$ & $2.36(0.60-9.23)$ \\
\hline \multicolumn{3}{|l|}{ Nutrient intake, $(n)$} \\
\hline Fat $(E \%) \geq 20$ and $<25$ & $0.87(0.32-2.37)$ & $0.48(0.18-1.26)$ \\
\hline Carbohydrate $(\mathrm{E} \%) \geq 50$ and $<70$ & $1.24(0.28-5.52)$ & $0.59(0.09-3.84)$ \\
\hline Dietary fiber $\geq 10 \mathrm{~g} / 1,000 \mathrm{kcal}$ & $3.19(0.70-14.57)$ & $1.01(0.22-4.70)$ \\
\hline Salt $<4.5 \mathrm{~g} / 1,000 \mathrm{kcal}$ & $0.43(0.16-1.16)$ & $0.70(0.27-1.85)$ \\
\hline Females, $n$ & 174 vs. 120 & 174 vs. 120 \\
\hline \multicolumn{3}{|l|}{ Physical activity } \\
\hline Regular exerciser & $2.38(1.35-4.19)$ & $1.11(0.64-1.92)$ \\
\hline Walking steps $\geq 6,000 /$ day & $4.17(2.10-8.27)$ & $1.88(0.97-3.64)$ \\
\hline \multicolumn{3}{|l|}{ Dietary behavior } \\
\hline Eating slowly, yes & $1.70(0.95-3.02)$ & $1.27(0.71-2.28)$ \\
\hline Not eating until full, yes & $1.47(0.79-2.73)$ & $2.95(1.45-5.99)$ \\
\hline Habitually eating snacks, no & $0.97(0.55-1.71)$ & $0.95(0.55-1.66)$ \\
\hline Not Habitual drinker & $0.79(0.37-1.71)$ & $0.88(0.42-1.82)$ \\
\hline \multicolumn{3}{|l|}{ Nutrient intake, $(n)$} \\
\hline Fat $(\mathrm{E} \%) \geq 20$ and $<25$ & $2.39(1.26-4.55)$ & $1.95(1.04-3.66)$ \\
\hline Carbohydrate $(\mathrm{E} \%) \geq 50$ and $<70$ & $2.93(1.04-8.25)$ & $1.82(0.69-4.83)$ \\
\hline Dietary fiber $\geq 10 \mathrm{~g} / 1,000 \mathrm{kcal}$ & $2.12(1.12-4.03)$ & $1.39(0.74-2.63)$ \\
\hline Salt $<4.5 \mathrm{~g} / 1,000 \mathrm{kcal}$ & $1.16(0.64-2.08)$ & $1.18(0.64-2.16)$ \\
\hline All, $n$ & 213 vs. 184 & 213 vs. 184 \\
\hline \multicolumn{3}{|l|}{ Physical activity } \\
\hline Regular exerciser & $2.89(1.75-4.76)^{b}$ & $1.30(0.81-2.08)^{\mathrm{b}}$ \\
\hline Walking steps $\geq 6,000 /$ day & $3.80(2.07-6.97)^{\mathrm{b}}$ & $1.90(1.07-3.37)^{\mathrm{b}}$ \\
\hline \multicolumn{3}{|l|}{ Dietary behavior } \\
\hline Eating slowly, yes & $1.49(0.91-2.43)^{\mathrm{b}}$ & $1.24(0.75-2.04)^{\mathrm{b}}$ \\
\hline Not eating until full, yes & $1.51(0.88-2.61)^{\mathrm{b}}$ & $2.32(1.29-4.18)^{\mathrm{b}}$ \\
\hline Habitually eating snacks, no & $0.84(0.51-1.38)^{\mathrm{b}}$ & $1.05(0.66-1.68)^{\mathrm{b}}$ \\
\hline Not Habitual drinker & $0.83(0.42-1.63)^{b}$ & $1.08(0.57-2.03)^{\mathrm{b}}$ \\
\hline \multicolumn{3}{|l|}{ Nutrient intake, $(n)$} \\
\hline Fat $(\mathrm{E} \%) \geq 20$ and $<25$ & $1.71(1.03-2.85)^{\mathrm{b}}$ & $1.27(0.77-2.09)^{\mathrm{b}}$ \\
\hline Carbohydrate $(\mathrm{E} \%) \geq 50$ and $<70$ & $2.24(0.95-5.29)^{\mathrm{b}}$ & $1.30(0.55-3.06)^{\mathrm{b}}$ \\
\hline Dietary fiber $\geq 10 \mathrm{~g} / 1,000 \mathrm{kcal}$ & $2.17(1.20-3.90)^{\mathrm{b}}$ & $1.35(0.75-2.42)^{\mathrm{b}}$ \\
\hline Salt $<4.5 \mathrm{~g} / 1,000 \mathrm{kcal}$ & $0.90(0.55-1.46)^{\mathrm{b}}$ & $1.02(0.62-1.67)^{\mathrm{b}}$ \\
\hline
\end{tabular}

OR, Odds ratio; 95\%CI, 95\% confidence interval; E\%, Energy percent; g/1,000 kcal, g per 1,000 kcal energy intake.

a. Baseline category and age category were adjusted to obtain the multivariate odds ratio, unless otherwise specified.

b. Baseline category, age category and sex were adjusted to obtain the multivariate odds ratio. 


\section{Changes in cardiovascular disease risks}

Table 5 shows a comparison of changes in cardiovascular disease risks between the intervention group and the control group among subjects with each risk at baseline. In females, the average Framingham risk score in the intervention group was significantly decreased at 15 months $(P=$ $0.005)$ and showed a significantly smaller increase at 27 months $(P<0.001)$. The average decrease in weight among female subjects showing overweight at baseline in the intervention group was significantly decreased only at 27 months $(P=0.008)$. The average decrease in SBP among female subjects with hypertension risk at baseline in the intervention group was significantly decreased only at 27 months $(P=0.026)$. Although the average $\mathrm{HbA}_{1 \mathrm{c}}$ among female subjects with diabetes risk at baseline in the intervention group was significantly decreased at 15 months $(P=0.041)$ and 27 months $(P=0.002)$. In males, the average increase in TG in the intervention group was significantly smaller at 15 months $(P=0.002)$ and at 27 months $(P<0.001)$.

Table 6 shows the odds ratios of cardiovascular disease risks in the intervention group versus the control group. Multiple logistic regression analysis adjusted for each baseline risk category and age category showed that the proportions of females with hypertension risk or diabetes risk were significantly lower in the intervention group than those in the control group only at 15 months (OR 0.48, CI 0.27-0.85 and OR 0.27 , CI $0.08-0.85$, respectively) and the proportions of females who were overweight or showed dyslipidemia risk were significantly lower in the intervention group only at 27 months (OR 0.29, CI 0.10-0.82 and OR 0.39, CI $0.16-0.92$, respectively). Multiple logistic regression analysis adjusted for sex, each baseline risk category and age category showed that the proportion of those at hypertension risk was significantly lower in the intervention group than in the control group only at 15 months (OR 0.54 , CI 0.33-0.88), the proportion of those who were overweight or showed dyslipidemia risk was significantly lower in the intervention group only at 27 months (OR 0.43, CI 0.20-0.94 and OR 0.43 , CI $0.21-0.87$, respectively). The proportion of those showing diabetes risk was significantly decreased in the intervention group at both 15 month (OR 0.42, CI 0.18-0.97) and 27 months (OR 0.56, CI 0.32-0.99).

\section{Discussion}

There was a significant increase in those walking a preferable number of steps and significant reductions in diabetes risk in the intervention group compared with the control group both evaluation at 15 months and 27 month. The Framingham risk score was significantly decreased at 15 months and 27 months among females. Only at 27 months, there were significant reductions of overweight and dyslipidemia risk in the intervention group. To our knowledge, this is the first report showing the effect of a longterm lifestyle intervention in a Japanese community setting for the reduction of cardiovascular disease risks. The present study indicated the importance of a long-term approach, showing a significant reduction in subjects who were overweight at 27 months but not at 15 months. The program in this study required a relatively high level of manpower and cost. Therefore, from the perspective of cost-effectiveness, it remains to be shown whether the implementation of this program should be recommended for wider use. Examination to develop more effective programs is warranted.

In the present study, the average weight loss in the intervention group among females was $2.1 \mathrm{~kg}(3.4 \%)$ at 15 months and $2.5 \mathrm{~kg}(4.1 \%)$ at 27 month. This weight loss degree is congruent with literature on another lifestyle intervention program based on the "Health-Up Model Project" (Noda et al. 2006). Noda et al. (2006) reported a 6-month weight loss program for 155 overweight subjects, which included dietary advice, monthly individual health education and exercise in sports facilities or at home. One hundred and twenty-one subjects who participated in this program experienced a mean weight loss of almost $2.4 \mathrm{~kg}$ (3.4\%). Based on these data, it is speculated that these approaches to lifestyle modification were effective in promoting weight loss among overweight subjects and the long-term impact of lifestyle intervention on prevention of gaining weight back was assessed in this study.

Several previous studies have shown the long-term effect of lifestyle intervention on health outcome (Lindstrom et al. 2006; Li et al. 2008). The extended follow-up of the Finnish Diabetes Prevention Study, one of the best known of these intervention studies, has shown that intensive lifestyle intervention in people at high risk for type 2 diabetes resulted in sustained lifestyle change and a reduction in diabetes incidence, which persisted for a median of 3 years after individual lifestyle counselling stopped (Lindstrom et al. 2006). Although none of the report had a large sample size, there are a few reports available regarding the effect of the lifestyle intervention after the end of such programs in Japanese communities. Iso et al. (2002) demonstrated that an intensive community-based program was effective in reducing serum total cholesterol level non-pharmacologically during the first year and also reduced the likelihood of progressive worsening of hypercholesterolemia during the subsequent 8 years in a randomized controlled trial with 51 subjects in the intervention group and 53 subjects in the control group. Although our findings indicated that the 27-month lifestyle modification program in the intervention group was effective for dyslipidemia risk compared to that in the control group, continuous evaluation some time after the end of the program is needed to properly evaluate the effect of the program.

At baseline, the results for the number of walking steps was not in accordance with those indicating regular exercise: the proportion of those with a habit of regular exercise was higher in the intervention group than in the control group, but the proportion of those with a habit of walking a preferable number of steps was lower in the intervention group than in the control group. These findings might be safely interpreted by considering that subjects in the control 
Table 5. Comparison of changes in cardiovascular disease risks between intervention and control group among subjects with each risk at baseline.

\begin{tabular}{|c|c|c|c|c|c|c|c|}
\hline & & \multicolumn{3}{|c|}{ Males } & \multicolumn{3}{|c|}{ Females } \\
\hline & & Intervention & Control & \multirow{2}{*}{$P$-value ${ }^{\mathrm{c}}$} & Intervention & Control & \multirow{2}{*}{$P$-value ${ }^{\mathrm{c}}$} \\
\hline & & Mean \pm S.D. & Mean \pm S.D. & & Mean \pm S.D. & Mean \pm S.D. & \\
\hline Overweight ${ }^{\mathrm{e}}$ & $n$ & 18 & 19 & & 64 & 28 & \\
\hline \multirow[t]{3}{*}{ Weight, kg } & baseline & $74.6 \pm 6.9$ & $72.5 \pm 10.4$ & $0.468^{\mathrm{d}}$ & $61.7 \pm 7.1$ & $64.3 \pm 7.5$ & $0.112^{\mathrm{d}}$ \\
\hline & 15 months & $-1.7 \pm 2.1$ & $-0.6 \pm 2.2$ & 0.914 & $-2.1 \pm 2.4$ & $-1.7 \pm 4.1$ & 0.073 \\
\hline & 27 months & $-1.6 \pm 3.8$ & $-0.4 \pm 2.2$ & 0.753 & $-2.5 \pm 3.4$ & $-1.0 \pm 3.6$ & 0.008 \\
\hline \multirow[t]{3}{*}{ BMI, $\mathrm{kg} / \mathrm{m}^{2}$} & baseline & $26.9 \pm 1.7$ & $26.9 \pm 2.6$ & $0.885^{\mathrm{d}}$ & $27.4 \pm 2.3$ & $27.6 \pm 2.6$ & $0.609^{d}$ \\
\hline & 15 months & $-0.6 \pm 0.7$ & $-0.4 \pm 1.1$ & 0.767 & $-1.0 \pm 1.1$ & $-0.8 \pm 1.5$ & 0.437 \\
\hline & 27 months & $-0.5 \pm 1.3$ & $-0.3 \pm 1.1$ & 0.551 & $-1.0 \pm 1.4$ & $-0.5 \pm 1.5$ & 0.137 \\
\hline Hypertension risk ${ }^{\mathrm{f}}$ & $n^{\mathrm{b}}$ & 28 & 42 & & 103 & 69 & \\
\hline \multirow[t]{3}{*}{ SBP, mmHg } & baseline & $143.9 \pm 6.4$ & $142.2 \pm 9.6$ & $0.405^{\mathrm{d}}$ & $144.4 \pm 9.7$ & $143.7 \pm 9.9$ & $0.648^{\mathrm{d}}$ \\
\hline & 15 months & $-7.7 \pm 10.9$ & $-3.4 \pm 13.7$ & 0.868 & $-10.9 \pm 12.1$ & $-5.4 \pm 12.7$ & 0.063 \\
\hline & 27 months & $-1.8 \pm 11.8$ & $-0.8 \pm 11.3$ & 0.948 & $-11.2 \pm 14.1$ & $-8.0 \pm 15.3$ & 0.026 \\
\hline \multirow[t]{3}{*}{$\mathrm{DBP}, \mathrm{mmHg}$} & baseline & $84.0 \pm 7.8$ & $85.9 \pm 9.4$ & $0.384^{\mathrm{d}}$ & $83.2 \pm 8.4$ & $83.3 \pm 8.6$ & $0.931^{\mathrm{d}}$ \\
\hline & 15 months & $-1.5 \pm 6.2$ & $-0.6 \pm 8.3$ & 0.149 & $-3.3 \pm 8.0$ & $-1.6 \pm 8.1$ & 0.458 \\
\hline & 27 months & $+0.4 \pm 5.5$ & $+0.2 \pm 8.8$ & 0.098 & $-4.4 \pm 7.5$ & $-4.4 \pm 8.2$ & 0.579 \\
\hline Dyslipidemia risk $^{g}$ & $n^{\mathrm{b}}$ & 36 & 49 & & 148 & 92 & \\
\hline \multirow[t]{3}{*}{$\mathrm{TC}, \mathrm{mg} / \mathrm{dl}$} & baseline & $209.6 \pm 23.4$ & $214.4 \pm 22.7$ & $0.349^{\mathrm{d}}$ & $235.8 \pm 31.2$ & $236.3 \pm 25.2$ & $0.911^{\mathrm{d}}$ \\
\hline & 15 months & $-0.5 \pm 20.2$ & $+0.3 \pm 20.7$ & 0.171 & $-3.7 \pm 20.6$ & $+0.0 \pm 22.7$ & 0.167 \\
\hline & 27 months & $+8.9 \pm 21.9$ & $+7.8 \pm 26.4$ & 0.124 & $+1.8 \pm 23.1$ & $+10.7 \pm 29.8$ & 0.009 \\
\hline \multirow[t]{3}{*}{ HDL-C, mg/dl } & baseline & $54.6 \pm 14.5$ & $53.2 \pm 14.7$ & $0.657^{\mathrm{d}}$ & $66.6 \pm 15.5$ & $66.9 \pm 13.6$ & $0.856^{\mathrm{d}}$ \\
\hline & 15 months & $-0.6 \pm 5.2$ & $-0.7 \pm 5.9$ & 0.531 & $-0.8 \pm 7.0$ & $+1.5 \pm 7.4$ & 0.182 \\
\hline & 27 months & $+4.8 \pm 6.9$ & $+0.4 \pm 6.3$ & 0.148 & $+3.7 \pm 8.4$ & $+4.8 \pm 7.4$ & 0.113 \\
\hline \multirow[t]{3}{*}{ LDL-C, mg/dl } & baseline & $138.9 \pm 21.1$ & $135.8 \pm 23.0$ & $0.528^{\mathrm{d}}$ & $153.0 \pm 31.9$ & $152.7 \pm 24.0$ & $0.940^{\mathrm{d}}$ \\
\hline & 15 months & $-12.4 \pm 14.6$ & $-10.2 \pm 17.8$ & 0.612 & $-15.1 \pm 17.8$ & $-13.3 \pm 19.5$ & 0.511 \\
\hline & 27 months & $-0.7 \pm 19.3$ & $-0.5 \pm 24.1$ & 0.576 & $-7.2 \pm 21.2$ & $-1.4 \pm 26.2$ & 0.135 \\
\hline \multirow[t]{3}{*}{$\mathrm{TG}, \mathrm{mg} / \mathrm{dl}$} & baseline & $107.8 \pm 42.3$ & $131.4 \pm 72.0$ & $0.061^{\mathrm{d}}$ & $96.9 \pm 46.8$ & $99.7 \pm 41.7$ & $0.636^{\mathrm{d}}$ \\
\hline & 15 months & $+2.2 \pm 32.1$ & $+8.5 \pm 49.1$ & 0.002 & $+9.0 \pm 109.9$ & $+1.9 \pm 45.0$ & 0.747 \\
\hline & 27 months & $+1.6 \pm 34.7$ & $+20.0 \pm 34.1$ & $<0.001$ & $+1.0 \pm 39.6$ & $+3.5 \pm 40.0$ & 0.976 \\
\hline Diabetes risk $^{\mathrm{h}}$ & $n^{\mathrm{b}}$ & 7 & 19 & & 34 & 19 & \\
\hline \multirow[t]{3}{*}{ FPG, mg/dl } & baseline & $114.6 \pm 15.9$ & $116.0 \pm 13.5$ & $0.821^{\mathrm{d}}$ & $111.7 \pm 14.1$ & $113.6 \pm 19.3$ & $0.674^{\mathrm{d}}$ \\
\hline & 15 months & $+0.4 \pm 7.4$ & $-2.2 \pm 11.5$ & 0.916 & $-5.4 \pm 11.7$ & $-3.3 \pm 19.8$ & 0.420 \\
\hline & 27 months & $+0.6 \pm 6.8$ & $-0.6 \pm 15.8$ & 0.936 & $-6.5 \pm 11.1$ & $+0.7 \pm 25.8$ & 0.066 \\
\hline \multirow[t]{3}{*}{$\mathrm{HbA}_{1 \mathrm{c}}, \%$} & baseline & $5.86 \pm 0.57$ & $5.77 \pm 0.70$ & $0.768^{\mathrm{d}}$ & $5.75 \pm 0.39$ & $5.98 \pm 0.56$ & $0.083^{\mathrm{d}}$ \\
\hline & 15 months & $-0.07 \pm 0.17$ & $-0.02 \pm 0.17$ & 0.807 & $-0.03 \pm 0.19$ & $-0.10 \pm 0.48$ & 0.041 \\
\hline & 27 months & $+0.17 \pm 0.17$ & $+0.29 \pm 0.33$ & 0.879 & $+0.10 \pm 0.30$ & $+0.29 \pm 0.72$ & 0.002 \\
\hline \multirow[t]{4}{*}{ Framingam risk score, score } & $n$ & 39 & 64 & & 174 & 120 & \\
\hline & baseline & $12.2 \pm 2.7$ & $12.3 \pm 2.9$ & $0.818^{\mathrm{d}}$ & $14.3 \pm 2.8$ & $14.2 \pm 2.8$ & $0.858^{\mathrm{d}}$ \\
\hline & 15 months & $+0.1 \pm 1.4$ & $+0.3 \pm 1.0$ & 0.834 & $-0.3 \pm 1.6$ & $-0.1 \pm 2.0$ & 0.005 \\
\hline & 27 months & $-0.1 \pm 1.4$ & $+0.4 \pm 1.6$ & 0.751 & $+0.1 \pm 1.9$ & $+0.4 \pm 2.1$ & $<0.001$ \\
\hline
\end{tabular}

BMI, Body mass index; SBP, Systolic blood pressure; DBP, Diastolic blood pressure; TC, Total cholesterol; HDL-C, High-density lipoprotein cholesterol; LDL-C, Low-density lipoprotein cholesterol; TG, Triglycerides; FPG, Fasting plasma glucose; HbA 1 , Hemoglobin $\mathrm{A}_{1 \mathrm{c}}$.

a. Values are shown as mean \pm S.D., unless otherwise specified.

b. Number of subjects for this analysis after we excluded subjects who self-reported that they were taking each medication prescribed for hypertension, dyslipidemia and diabetes for 27 months.

c. $P$ value was determined by analysis of variance for repeated measures adjusted for age, unless otherwise specified.

d. $P$ value was determined by Student's $t$-test.

e. Subjects with overweight were defined as having a BMI $\geq 25 \mathrm{~kg} / \mathrm{m}^{2}$.

f. Subjects with hypertension risk were defined as having at least one of the following: $\mathrm{SBP} \geq 130 \mathrm{mmHg}$ or DBP $\geq 85 \mathrm{mmHg}$.

g. Subjects with dyslipidemia risk were defined as having at least one of the following: $\mathrm{TC} \geq 200 \mathrm{mg} / \mathrm{dl}$ (females aged 50 years or over: $220 \mathrm{mg} / \mathrm{dl}$ ), HDL-C $<40 \mathrm{mg} / \mathrm{dl}$, LDL-C $\geq 140 \mathrm{mg} / \mathrm{dl}$ or TG $\geq 150 \mathrm{mg} / \mathrm{dl}$.

h. Subjects with diabetes risk were defined as having at least one of the following: $\mathrm{FPG} \geq 110 \mathrm{mg} / \mathrm{dl}$ or $\mathrm{HbA}_{1 \mathrm{c}} \geq 5.6 \%$. 
Table 6. Odd ratios of cardiovascular disease risks in intervention vs. control group.

\begin{tabular}{|c|c|c|}
\hline & Intervention vs. Control at 15 months & Intervention vs. Control at 27 months \\
\hline & OR $(95 \% C I)^{a}$ & OR $(95 \% C I)^{a}$ \\
\hline Males, $n$ & 39 vs. 64 & 39 vs. 64 \\
\hline Overweight $^{\mathrm{c}}$ & $1.12(0.27-4.63)$ & $0.72(0.18-2.83)$ \\
\hline Hypertension risk $^{\mathrm{d}}$ & $0.70(0.26-1.86)$ & $0.74(0.22-2.47)$ \\
\hline Dyslipidemia risk ${ }^{\mathrm{e}}$ & $0.31(0.09-1.11)$ & $0.42(0.11-1.57)$ \\
\hline Diabetes risk ${ }^{\mathrm{f}}$ & $0.72(0.19-2.74)$ & $0.72(0.23-2.33)$ \\
\hline Females, $n$ & 174 vs. 120 & 174 vs. 120 \\
\hline Overweight $^{\mathrm{c}}$ & $0.37(0.13-1.02)$ & $0.29(0.10-0.82)$ \\
\hline Hypertension risk ${ }^{\mathrm{d}}$ & $0.48(0.27-0.85)$ & $0.79(0.47-1.34)$ \\
\hline Dyslipidemia risk $^{\mathrm{e}}$ & $0.61(0.31-1.21)$ & $0.39(0.16-0.92)$ \\
\hline Diabetes risk ${ }^{\mathrm{f}}$ & $0.27(0.08-0.85)$ & $0.55(0.29-1.05)$ \\
\hline All, $n$ & 213 vs. 184 & 213 vs. 184 \\
\hline Overweight $^{\mathrm{c}}$ & $0.61(0.29-1.30)^{\mathrm{b}}$ & $0.43(0.20-0.94)^{\mathrm{b}}$ \\
\hline Hypertension risk ${ }^{\mathrm{d}}$ & $0.54(0.33-0.88)^{\mathrm{b}}$ & $0.79(0.49-1.28)^{\mathrm{b}}$ \\
\hline Dyslipidemia risk ${ }^{\mathrm{e}}$ & $0.59(0.33-1.06)^{\mathrm{b}}$ & $0.43(0.21-0.87)^{b}$ \\
\hline Diabetes risk $^{\mathrm{f}}$ & $0.42(0.18-0.97)^{\mathrm{b}}$ & $0.56(0.32-0.99)^{b}$ \\
\hline
\end{tabular}

OR, Odds ratio; 95\%CI, 95\% confidence interval; BMI, Body mass index; SBP, Systolic blood pressure; DBP, Diastolic blood pressure; TC, Total cholesterol; HDL-C, High-density lipoprotein cholesterol; LDL-C, Low-density lipoprotein cholesterol; TG, Triglycerides; FPG, Fasting plasma glucose; $\mathrm{HbA}_{1 \mathrm{c}}$, Hemoglobin $\mathrm{A}_{1 \mathrm{c}}$.

a. Baseline risk category and age category were adjusted to obtain the multivariate odds ratio, unless otherwise specified.

b. Baseline risk category, age category and sex were adjusted to obtain the multivariate odds ratio.

c. Subjects with overweight were defined as having a BMI $\geq 25 \mathrm{~kg} / \mathrm{m}^{2}$.

f. Subjects with hypertension risk were defined as having at least one of the following: $\mathrm{SBP} \geq 130 \mathrm{mmHg}$, $\mathrm{DBP} \geq 85 \mathrm{mmHg}$ or medication usage for hypertension.

g. Subjects with dyslipidemia risk were defined as having at least one of the following: $\mathrm{TC} \geq 200 \mathrm{mg} / \mathrm{dl}$ (females aged 50 years or over: $220 \mathrm{mg} / \mathrm{dl}$ ), HDL-C $<40 \mathrm{mg} / \mathrm{dl}$, LDL-C $\geq 140 \mathrm{mg} / \mathrm{dl}$, TG $\geq 150 \mathrm{mg} / \mathrm{dl}$ or medication usage for dyslipidemia.

h. Subjects with diabetes risk were defined as having at least one of the following: $\mathrm{FPG} \geq 110 \mathrm{mg} / \mathrm{dl}, \mathrm{HbA}$. $\geq 5.6 \%$ or medication usage for diabetes.

group walked more than the intervention group during daily life and on their jobs, but they did not recognize walking as exercise.

Our study includes several limitations. First, this study had a non-randomized study design: the participants were allocated to either the intervention or control group based on each participant's request. Especially among female participants, the proportion of those who were overweight was significantly higher in the intervention group than in the control group, so there is a possibility that subjects in the intervention group had more lifestyle style behaviors that should be modified compared with those in the control group. In addition, the possibility that the lack of masking for group allocation might have impacted participants' lifestyle behavior improvement over the program cannot be denied. Second, the measures of lifestyle were self-reported by participants, which might have resulted in some degree of measurement error. Third, the fact that 152 subjects ( $27.7 \%$ of baseline participants) dropped out over 27 months might have influenced the results because of the possibility that there was weaker motivation for lifestyle modification among those subjects. Fourth, the study participants were volunteers, so they were probably more health-conscious than the general population. Lastly, the number of male participants was relatively small, which might have limited the significance of differences in the results. Our findings should be generalized to middle-aged Japanese with caution because of possible selection bias.

In conclusion, the relatively intensive 27 -month intervention program is effective in improving lifestyle behavior and decreasing cardiovascular disease risks at the end of the program.

\section{Acknowledgments}

This work was supported grants from the Ministry of Health, Labour and Welfare of Japan [No1217003]. The authors thank all staff who working in the Health-Up Model Project and the citizens of Soka city, Saitama prefecture, Japan, who participated in this study.

\section{References}

Amano, N., Ogata, N., Morita, N., Saeki, K., Notani, M., Komukai, H., Azuma, Y., Matsuda, R. \& Kurumatani, N. (2002) Effects of nutrition education for residents on intake of lipid-related nutrients. Nippon Koshu Eisei Zasshi, 49, 332-343 (In Japanese).

Appel, L.J., Champagne, C.M., Harsha, D.W., Cooper, L.S., 
Obarzanek, E., Elmer, P.J., Stevens, V.J., Vollmer, W.M., Lin, P.H., Svetkey, L.P., Stedman, S.W. \& Young, D.R. (2003) Effects of comprehensive lifestyle modification on blood pressure control: main results of the PREMIER clinical trial. JAMA, 289, 2083-2093.

Committee on Guideline for the Management of Hypertension. (2009) Guideline for the management of hypertension. The Japanese society of hypertension, Tokyo (In Japanese).

Committee on Guideline for Treatment of Obesity. (2006) A guideline for treatment of obesity 2006. Japan Society for the Study of Obesity, Tokyo (In Japanese).

Egawa, K., Oida, Y., Arao, T. \& Matsuzuki, H. (2004) Preliminary study for development of a prevention program for life-style related diseases using a health examination in community. Tairyoku Kenkyu, 102, 15-29 (In Japanese).

Egawa, K., Oida, Y., Arao, T., Matsuzuki, H., Shirako, M. \& Kasai, W. (2007) Efficacy of a community-based weight reduction program to improve exercise and diet behavior in overweight adults. Nippon Koshu Eisei Zasshi, 54, 847-856 (In Japanese).

Elmer, P.J., Obarzanek, E., Vollmer, W.M., Simons-Morton, D., Stevens, V.J., Young, D.R., Lin, P.H., Champagne, C., Harsha, D.W., Svetkey, L.P., Ard, J., Brantley, P.J., Proschan, M.A., Erlinger, T.P. \& Appel, L.J. (2006) Effects of comprehensive lifestyle modification on diet, weight, physical fitness, and blood pressure control: 18-month results of a randomized trial. Ann. Intern. Med., 144, 485-495.

Expert Panel on Detection, Evaluation, and Treatment of High Blood Cholesterol in Adults. (2001) Executive summary of the third report of the national cholesterol education program (NCEP) expert panel on detection, evaluation, and treatment of high blood cholesterol in adults (Adult Treatment Panel III). JAMA, 285, 2486-2497.

Gillies, C.L., Abrams, K.R., Lambert, P.C., Cooper, N.J., Sutton, A.J., Hsu, R.T. \& Khunti, K. (2007) Pharmacological and lifestyle interventions to prevent or delay type 2 diabetes in people with impaired glucose tolerance: systematic review and metaanalysis. BMJ, 334, 299.

Haruyama, Y., Muto, T., Nakade, M., Kobayashi, E., Ishisaki, K. \& Yamasaki, A. (2009) Fifteen-month lifestyle intervention program to improve cardiovascular risk factors in a community population in Japan. Tohoku J. Exp. Med., 217, 259-269.

Health and Welfare Statistic Association. (2008) Journal of health and welfare statistics, 55, 82-83 (In Japanese).

Iso, H., Imano, H., Nakagawa, Y., Kiyama, M., Kitamura, A., Sato, S., Naito, Y., Shimamoto, T. \& Iida, M. (2002) One-year community-based education program for hypercholesterolemia in middle-aged Japanese: a long-term outcome at 8-year followup. Atherosclerosis, 164, 195-202.

Iso, H., Shimamoto, T., Yokota, K., Sankai, T., Jacobs, D.R., Jr. \& Komachi, Y. (1996) Community-based education classes for hypertension control. A 1.5-year randomized controlled trial. Hypertension, 27, 968-974.

Japan Atherosclerosis Society. (2007) Japan atherosclerosis society guidelines for prevention of atherosclerotic cardiovascular diseases, 2007. Japan Atherosclerosis Society, Tokyo (In Japanese).

Japan Diabetes Society. (2008) Guide for diabetes care 2008-2009. Bunkodo Corp., Tokyo (In Japanese).

Japan Public Health Association. (2002) Guideline of Diabetes and Cardiovascular Diseases in Health Care of Aged. (Accessed February 24, 2010, at http://www.jpha.or.jp) (In Japanese)

Knowler, W.C., Barrett-Connor, E., Fowler, S.E., Hamman, R.F., Lachin, J.M., Walker, E.A., Nathan, D.M. (2002) Reduction in the incidence of type 2 diabetes with lifestyle intervention or metformin. N. Engl. J. Med., 346, 393-403.

Kuriyama, S., Shimazu, T., Hozawa, A., Yabe, M., Tasaki, M., Mononaga, Y., Sakai, M., Miura, C., Ito, F., Ito, T., Yabe, H., Nitta, S., Suzuki, R., Fujita, K., Nagatomi, R. \& Tsuji, I. (2006) Comparison of intensive and moderate individual life-style intervention programs for overweight or obese persons with fasting glucose levels of $95-125 \mathrm{mg} / \mathrm{dl}$ in Japan. Nippon Koshu Eisei Zasshi, 53, 122-132 (In Japanese).

Li, G., Zhang, P., Wang, J., Gregg, E.W., Yang, W., Gong, Q., Li, H., Jiang, Y., An, Y., Shuai, Y., Zhang, B., Zhang, J., Thompson, T.J., Gerzoff, R.B., Roglic, G.Hu, Y. \& Bennett, P.H. (2008) The long-term effect of lifestyle interventions to prevent diabetes in the China Da Qing Diabetes Prevention Study: a 20-year follow-up study. Lancet, 371, 1783-1789.

Lindstrom, J., Ilanne-Parikka, P., Peltonen, M., Aunola, S., Eriksson, J.G., Hemio, K., Hamalainen, H., Harkonen, P., Keinanen-Kiukaanniemi, S., Laakso, M., Louheranta, A., Mannelin, M., Paturi, M., Sundvall, J., Valle, T.T., Uusitupa, M. \& Tuomilehto, J. (2006) Sustained reduction in the incidence of type 2 diabetes by lifestyle intervention: follow-up of the Finnish Diabetes Prevention Study. Lancet, 368, 1673-1679.

Lindstrom, J., Louheranta, A., Mannelin, M., Rastas, M., Salminen, V., Eriksson, J., Uusitupa, M. \& Tuomilehto, J. (2003) The Finnish Diabetes Prevention Study (DPS): Lifestyle intervention and 3-year results on diet and physical activity. Diabetes Care, 26, 3230-3236.

Maruthur, N.M., Wang, N.Y. \& Appel, L.J. (2009) Lifestyle interventions reduce coronary heart disease risk: results from the PREMIER Trial. Circulation, 119, 2026-2031.

Maruyama, K., Sato, S., Ohira, T., Maeda, K., Noda, H., Kubota, Y., Nishimura, S., Kitamura, A., Kiyama, M., Okada, T., Imano, H., Nakamura, M., Ishikawa, Y., Kurokawa, M., Sasaki, S. \& Iso, H. (2008) The joint impact on being overweight of self reported behaviours of eating quickly and eating until full: cross sectional survey. $B M J, \mathbf{3 3 7}$, a2002.

Ministry of Education, Culture, Sports, Science and Technology, and Ministry of Health, Labour and Welfare, Japan. (2002) Ethical Guidelines for Epidemiological Research. (Accessed March 25, 2010, at http://www.niph.go.jp/wadai/ekigakurinri/ index.htm) (In Japanese).

Ministry of Health, Labour and Welfare of Japan. (2004) Report on the survey of diabetes 2002. (Accessed December 27, 2009, at http://www.mhlw.go.jp/toukei/kouhyo/indexkk_4_2.html) (In Japanese).

Ministry of Health, Labour and Welfare of Japan. (2005a) Kokuho health-up project, manual for indiviudal health support program ver.1. All-Japan Federation of National Health Insurance Organizations, Tokyo (In Japanese).

Ministry of Health, Labour and Welfare of Japan. (2005b) Dietary reference intake for Japanese, 2005. Daiichi Shuppan Publishing Co., Ltd., Tokyo (In Japanese).

Ministry of Health, Labour and Welfare of Japan. (2009) The National Health and Nutrition Survey in Japan, 2006. Daiichi Shuppan publishing, Co., Ltd., Tokyo (In Japanese).

Mitsuhashi, E., Lee, J.S. \& Kawakubo, K. (2003) Body weight fluctuation and life-style change after short-term weight reduction programs. Nippon Koshu Eisei Zasshi, 50, 136-145 (In Japanese).

Miura, K., Myogadani, H., Kadoya, Y., Hayashi, M., Motoya, M., Kuzumaki, M., Yoneda, M., Mitsui, T., Nishijo, M., Morikawa, Y., Nakanishi, Y., Nakashima, M. \& Nakagawa, H. (2006) Effectiveness of lifestyle modification programs for control of blood pressure: a non-randomized controlled trial in Komatsu, Japan. Nippon Koshu Eisei Zasshi, 53, 533-542 (In Japanese).

National Cholesterol Education Program (NCEP) Expert Panel on Detection, Evaluation, and Treatment of High Blood Cholesterol in Adults (Adult Treatment Panel III). (2002) Third Report of the National Cholesterol Education Program (NCEP) Expert Panel on Detection, Evaluation, and Treatment of High Blood Cholesterol in Adults (Adult Treatment Panel III) final report. Circulation, 106, 3143-3421.

Noda, H., Harada, M., Yokota, K., Umesawa, M., Yamagishi, K., Cui, R., Ikeda, A., Chei, C., Wakabayashi, Y., Inagawa, M., Toriumi, S., Hirose, K., Oshima, M., Shiina, Y., Tanigawa, T., 
Tanaka, K., Shimamoto, T. \& Iso, H. (2006) Individualized health education with sports gym use and dietary advice for overweight and obese persons in a community. Kokuho Health-up model Program in Chikusei-shi (former Kyowa town). Nippon Koshu Eisei Zasshi, 53, 749-761 (In Japanese).

Otsuka, R., Tamakoshi, K., Yatsuya, H., Murata, C., Sekiya, A., Wada, K., Zhang, H.M., Matsushita, K., Sugiura, K., Takefuji, S., OuYang, P., Nagasawa, N., Kondo, T., Sasaki, S. \& Toyoshima, H. (2006) Eating fast leads to obesity: findings based on self-administered questionnaires among middle-aged Japanese men and women. J. Epidemiol., 16, 117-124.

Otsuka, R., Tamakoshi, K., Yatsuya, H., Wada, K., Matsushita, K., OuYang, P., Hotta, Y., Takefuji, S., Mitsuhashi, H., Sugiura, K., Sasaki, S., Kral, J.G. \& Toyoshima, H. (2008) Eating fast leads to insulin resistance: findings in middle-aged Japanese men and women. Prev. Med., 46, 154-159.

Ratner, R., Goldberg, R., Haffner, S., Marcovina, S., Orchard, T., Fowler, S. \& Temprosa, M. (2005) Impact of intensive lifestyle and metformin therapy on cardiovascular disease risk factors in the diabetes prevention program. Diabetes Care, 28, 888-894.

Sairenchi, T., Iso, H., Irie, F., Fukasawa, N., Yamagishi, K.,
Kanashiki, M., Saito, Y., Ota, H. \& Nose, T. (2005) Age-specific relationship between blood pressure and the risk of total and cardiovascular mortality in Japanese men and women. Hypertens. Res., 11, 901-909.

Sakuma, I. \& Kitabatake, A. (2008) Guideline for primary prevention of ischemic heart disease. Nippon Rinsyo, 66, 319-324 (In Japanese).

Sasaki, S. (2007) Dietary reference intake for Japanese (2005) (The report from the scientific committee of "Dietary reference intake for Japanese- recommended dietary allowance-). (Accessed December 27, 2009, at http://www.nih.go.jp/eiken/ english/research/program_epidemiology.html).

Takahashi, K. (2003) Food frequency questionnaire based on food groups for estimating individual nutrient intake. Eiyougaku Zasshi, 61, 161-169 (In Japanese).

Woo, J., Cheung, B., Ho, S., Sham, A. \& Lam, T.H. (2008) Influence of dietary pattern on the development of overweight in a Chinese population. Eur. J. Clin. Nutr., 62, 480-487.

Yoshimura, Y. \& Takahashi, K. (2001) Excel eiyoukun food frequency questionnaire. Kenpakusha Corp., Tokyo (In Japanese). 